\title{
Minireview
}

\section{Function and evolution of plastid sigma factors}

\author{
Takashi Shiina*, Yoko Ishizaki, Yusuke Yagi, Yoichi Nakahira \\ Graduate School of Life and Environmental Sciences, Kyoto prefectural University, Shimogamo, Sakyo-ku, Ktyoto 606- \\ 8522 Japan \\ *E-mail: shiina@kpu.ac.jp Tel \& Fax: +81-75-703-5449
}

Received November 20, 2008; accepted February 2, 2009 (Edited by K. Kanamaru)

\begin{abstract}
Chloroplasts are plant-specific organelles that perform photosynthesis and responsible for the world's primary productivity. Using light energy, chloroplasts produce many important products, including starch, amino acids, lipids, pigments and various secondary products. Therefore, chloroplasts are essential to the lives of all plants and animals alike. Chloroplast transformation is a unique technology to produce huge amount of valuable materials in chloroplasts using photosynthetic energy. In order to control chloroplasts at will, we need more information on molecular basis of chloroplast gene expression and communication between the chloroplast and nucleus. Chloroplast sigma factors are key regulators of the chloroplast gene expression and chloroplast differentiation. This review summarized recent findings on roles of chloroplast sigma factors in the chloroplast differentiation and environmental responses.
\end{abstract}

Key words: Chloroplast, plastid, sigma factor, transcription.

Plastids are unique plant organelles that exist in several different forms. Plastids are dynamic and can convert between types by external and developmental signals. The most common plastid type is the chloroplast responsible for photosynthesis in land plants, algae and some protists. All living things depend on energy produced from photosynthesis. Moreover, chloroplasts carry out other essential biochemical processes, including starch, amino acids, fatty acids and pigments synthesis, nitrogen and sulfate assimilation, hormone synthesis and various secondary metabolism. Not only plants but also human life is dependent on these important products from plastids.

Plastids are known to have originated from prokaryotic photosynthetic cyanobacteria. Consequently plastids are semi-autonomous and contain their own genetic system. In higher plants, plastids contain a small prokaryotic-type genome which encodes $\sim 120$ genes. Higher plant plastids contain two types of RNA polymerases, eubacterial-type PEP (plastid-encoded plastid RNA polymerase) and phage-type NEP (nuclearencoded plastid RNA polymerase) (Hess and Börner, 1999). In order to recognize promoters, PEP requires an exchangeable subunit, sigma factor. In bacteria, a variety of sigma factors specifically recognize different promoter sequences. The sigma factor determines which genes are transcribed. Higher plants also encode multiple sigma factor genes (Kanamaru and Tanaka, 2004). Recent molecular and genetic analyses revealed the roles of plastid sigma factors in chloroplast differentiation and environmental responses. In this review, we summarize plastid transcription and its regulation in higher plants, focused on plastid sigma factors.

\section{Plastid genomes}

Plastids have evolved from ancestral photosynthetic cyanobacteria, which were taken into the host cells as endosymbionts. It is believed that chloroplasts are monophyletic, with one primary endosymbiosis. Thus, plastids retain a lot of typical cyanobacterial features such as circular genome of 100-200 kbp and prokaryotictype gene expression system. During plant cell evolution, a large fraction of the original endosymbiont genes has either been lost or transferred to the nucleus. In consequence, the higher plant plastomes contain a limited number of conserved genes. For example, Arabidopsis chloroplast genome encodes only 129 genes, including 47 photosynthesis-related genes, 25 ribosomal genes, four RNA polymerase subunit genes, nine other protein coding genes (total 85 protein genes), 34 tRNA genes and 4 rRNA genes (Sato et al. 1999). On the other hand, chloroplast is believed to contain more than 3,000 proteins. Recent proteome analysis identified 1325 proteins in chloroplasts (Zybailov et al. 2008). More than $90 \%$ of them are encoded on nuclear DNA, synthesized in cytosol and transported into chloroplasts. Consequently, chloroplast function is largely dependent on the nuclei via nuclear-encoded proteins. It should be noted that DNA transfer from plastids to the nucleus is 
ongoing (Matsuo et al. 2005).

In contrast to photosynthesis-related genes and ribosome genes, which are retained on the plastid genomes, plastid genomes have selectively lost prokaryotic genes for transcription factors during evolution. For example, sigma factor genes that are involved in promoter recognition and transcription initiation of prokaryotic RNA polymerases have been transferred to the nucleus at an early stage of plant evolution (see below). No sigma factor gene has been identified in chloroplast genomes of extant plants. Moreover, plant cells have lost prokaryotic genes for DNA binding proteins during evolution. Cyanobacteria encodes $\sim 150$ transcription factors (Nakamura et al. 1998), but chloroplast genome of primitive red algae C. melolae encodes just four putative transcription factor genes (Matsuzaki et al. 2004). Moreover, no prokaryotic DNA binding protein gene has been identified in both chloroplast and nuclear genomes of higher plants, suggesting that plants have lost prokaryotic during plant evolution (Sato 2001). Instead of prokaryotic factors, plastids acquired eukaryotic transcription factors to control plastid gene expression. As a consequence, plastid transcription is highly dependent on the nuclearencoded sigma factors and eukaryotic type transcription factors in higher plants.

\section{Plastid RNA polymerases}

Cyanobacteria contain a multi subunit-type prokaryotic RNA polymerase (RNAP). Its catalytic core is composed of $2 \alpha, 1 \beta, 1 \beta^{\prime}$ and $1 \beta^{\prime \prime}$ subunits. Unlike other eubacteria, the $\beta^{\prime}$ subunit is usually split into two parts in cyanobacteria, $\beta^{\prime}$ and $\beta^{\prime \prime}$ subunits. The plastid genome contains rpo genes encoding homologs of the cyanobacterial RNAP $\alpha(r p o A), \beta$ (rpoB), $\beta^{\prime}$ (rpoCl) and $\beta^{\prime \prime}$ (rpoC2) subunits. This cyanobacterial-type RNAP is widely conserved in almost all of plant cells, except for achlorophyllous parasitic plant such as C. gronovii (Funk et al, 2007) and E. virginiana (Wolfe et al. 1992) The expected molecular masses for the PEP subunits of Arabidopsis are $38 \mathrm{kD}(\alpha), 121 \mathrm{kD}(\beta), 79 \mathrm{kD}\left(\beta^{\prime}\right)$, and $156 \mathrm{kD}\left(\beta^{\prime \prime}\right)$. These subunits form the core of the plastid-encoded plastid RNA polymerase (PEP). Sigma factors interact with PEP core and confer promoterspecific binding and transcription specificity. Higher plants contain multiple nuclear-encoded sigma factors, which direct RNA polymerase to distinct sets of promoters in response to environmental and developmental signals (see below).

One of the most unique points of the plastid transcription system is that the T7 phage type RNA polymerases (NEP) are working together with the prokaryotic type RNA polymerase PEP (Hess and Börner, 1999). In spite of small size of plastome, the transcription system of plastids is much more complex than that of prokaryotes. The drug sensitivity of NEP is different from bacterial-type RNA polymerase and similar to the phage T7 RNA polymerase. Arabidopsis contains two plastid-targeting phage-type NEP (RpoTp and RpoTmp), aside from mitochondrial RNA polymerase (RpoTm). It has been shown that RpoTp is primarily targeted to chloroplasts, while RpoTmp is dually targeted both into plastids and mitochondria (Hedtke et al, 2000). Plastid NEP genes might have derived from a gene duplication event of a mitochondrial NEP gene (RpoTm). Interestingly, plastid-target phagetype RNAP gene is absent in the green alga Chlamydomonas. On the other hand, two phagetype RNAP genes have been identified in moss Physcomitrella patens, suggesting that NEP has been evolved from RpoTm during the evolution of land plants (Kabeya et al. 2002).

\section{PEP and NEP}

Plastid-encoded genes are classified into three groups; type I photosynthesis genes that are transcribed only from PEP promoters, type II photosynthesis and housekeeping genes that are transcribed from both PEP and NEP promoters, and type III NEP-dependent genes, including PEP core subunit genes (rpoA, rpoB and $r p o C$ ), $a c c D$ (acetyl-CoA carboxylase) and $y c f 2$ (Hajdukiewicz et al. 1997; Shiina et al. 2005). It was generally assumed that during plastid differentiation the building up of the NEP transcription system precedes the building up of the PEP transcription system. At first NEP is activated and transcribes the type II and type III housekeeping genes involved in PEP construction, ribosome biogenesis and lipid synthesis. And then newly synthesized PEP takes over transcription during later stages. PEP actively transcribes type I and type II photosynthesis genes, including PSI and PSII subunits and rubisco large subunit in developing chloroplasts, and supports the quick building up of photosynthesis apparatus (Mullet, 1993). However, recent detailed expression studies revealed that both PEP and NEP transcription systems are built up simultaneously during germination (Demarsy et al. 2006). Both NEP and PEP proteins are present in dry seeds of Arabidopsis. NEP (RpoTp and RpoTmp) protein levels are transiently upregulated at an early phase of chloroplast development (2 days after germination), and subsequently decreased up to 5 days (Demarsy et al. 2006; Emanuel et al. 2006). Similarly, not only NEP but also PEP core protein levels are also up-regulated quickly during germination. Roles of plastid sigma factors in the switch from predominant NEP to predominant PEP activity during plant development will be discussed later. 


\section{Plastid promoters}

\section{Standard PEP promoters}

According to the presence of multiple RNA polymerases and multiple sigma factors (promoter recognition subunit of PEP), different types of promoters have been mapped on the plastid genomes. Standard PEP promoters resemble $E$. coli $\sigma^{70}$-type promoters that are characterized by consensus -35 and -10 sequences spaced at 17 19 nt. In fact, purified E. coli RNA polymerase holoenzyme is able to initiate transcription from the standard $\sigma^{70}$-type PEP promoters, suggesting that plastid PEP promoters share common features with eubacterial consensus promoters. Meanwhile, relative PEP promoter strength varies more than 40 times. The PEP promoter strength is probably determined by the sequence similarity of the promoter elements to consensus sequences. Usually, PEP transcription activity is transiently up-regulated during chloroplast development and declines significantly later in mature leaves. However, $p s b A$, $p s b D$, and $r r n P 1$ promoters remain active in mature chloroplasts. These PEP promoters exhibit unique promoter structures.

\section{The psbA promoter}

Plastid $p s b A$ encodes D1 protein of the photosystem II core complex. The $p s b A$ promoter is one of the most active PEP promoters in chloroplasts. Plant cells counteract photoinhibition by increasing D1 protein turn over. It is assumed that the $p s b A$ promoter is responsible for the differential maintenance of high transcription activity in mature leaves to support the light-dependent rapid turn over of D1 protein. The $p s b A$ promoter represents a typical $\sigma^{70}$-type PEP promoter, but contains two additional motifs; TATA box-like motif and TGn motif, which regulate the transcription initiation efficiency. The TATA box-like motif (TATATA) locating -24 and -19 nt of the transcription initiation site is well conserved among Characeae and land plants. In vitro and in vivo experiments suggested that the TATA box-like motif contributed to the $p s b A$ promoter activity in mature chloroplasts (Eisermann et al. 1990; Kim et al. 1999). The TGn motif is found upstream of the -10 element of the psbA promoter of many higher plants and forms an extended -10 promoter. In wheat the TGn motif is essential for the -35 element-independent $p s b A$ transcription (Satoh et al. 1999).

\section{The $p s b D$ light-responsive promoter}

The $p s b D$ encode another PSII reaction center protein D2 that also shows a light-dependent rapid turnover (Christopher and Mullet, 1994). The psbD lightresponsive promoter ( $p s b D$ LRP) is a unique PEP promoter, which is specifically activated by highirradiance blue and UV-A light (Christopher and Mullet,
1994). The psbD LRP also exhibited circadian rhythmicity (Nakahira et al. 1997). Chryptochromes and phytochrome A likely mediate blue light-dependent transcription from the $p s b D$ LRP (Thum et al. 2001; Chun et al. 2001). Furthermore, it should be noted that the $p s b D$ LRP is activated by various stresses, including low temperature, salt and osmotic stresses (Nagashima et al. 2004). Genome-wide analysis of plastome transcription in stressed plants revealed that the $p s b D$ LRP is the only plastid promoter whose activity is specifically regulated by environmental stresses (Kin et al. unpublished data).

The $p s b D$ LRP is characterized by a weakly conserved -35 element and a conserved 28-nt upstream sequence (AAG box: $-36 \sim-64 \mathrm{nt}$ ). Aside from the standard PEP promoters that are transcribed only by PEP holoenzyme, the $p s b D$ LRP activity is dependent on the upstream AAG-box and the AAG-box binding factors (AGF) in various plants (Kim and Mullet, 1995; To et al. 1996; Nakahira et al. 1998). The AAG-box is well conserved among gymnosperms and angiosperms, but ferns and moss have different types of upstream elements (Shimmura et al. unpublished data). PTF1 is a putative AGF that has been identified by yeast one hybrid screening (Baba et al. 2001). However, recent study revealed the nuclear localization of PTF1, suggesting its possible function as a nuclear transcription factor (Kodama and Sano, 2007). Recently, it has been revealed that light-dependent activation of the $p s b D$ LRP is mediated by a special sigma factor AtSIG5 (see below).

\section{The rrn P1promoter}

The $r r n$ P1 promoter is a major promoter in higher plants that is responsible for transcription of the rrn operon encoding four rRNA molecules and two tRNA genes. The rrn P1 promoter contains well conserved -35 and -10 elements, In addition to these consensus sequence elements, a hexameric sequence, named the rRNA operon upstream activator (RUA) has been identified just upstream of the -35 element (Suzuki et al. 2003). The RUA sequence is well conserved in higher plants. It has been shown that the RUA play a role in $r r n \mathrm{P} 1$ promoter activity, although it is not clear whether the RUA is recognized directly by PEP or by specific DNA-binding proteins.

\section{The NEP promoters}

Most NEP promoters share a 10-nt consensus sequence overlapping the transcription initiation site. These consensus-type NEP promoters (Type I) contain a conserved YRTA motif just upstream of the transcription initiation site. This feature is similar to mitochondrial and T3/T7 phage type promoters (Hajdukiewicz et al. 1997). On the other hand, a non-consensus type NEP promoter (Type II) has been identified upstream of $c l p P$ 
gene encoding the catalytic subunit of the ClpP ATPdependent protease (Sriraman et al. 1998). The clpP promoter lacks a YRTA motif, but its activity is dependent on downstream sequences $(-5$ to +25$)$ of the transcription initiation site.

Recent molecular genetic analyses of RpoTp and RpoTmp revealed that two NEP enzymes have overlapping as well as gene-specific functions in the transcription of plastid genes (Swiatecka-Hagenbruch et al. 2008; Courtois et al. 2008). RpoTp plays a major role in transcription of NEP promoters and RpoTmp might play a major role in transcription from the $\operatorname{clpP}$ and $r r n$ Pc NEP promoters.

\section{Functional characterization of plastid sigma factors}

Sigma factors interact with PEP to confer promoterspecific binding and transcription specificity. Bacterial cells usually contain several sigma factors with different functions and play a central role in transcriptional control of cell proliferation and environmental responses. The sigma factors form a large family, the $\sigma^{70}$ family, which can be divided into three groups with different structure and function (Lonetto et al. 1992). Under normal growth conditions, an RNA polymerase holoenzyme containing the principal sigma factors (Group 1) such as E. coli $\sigma^{70}$ play a major role in the transcription of housekeeping genes. These principal sigma factors are essential for cell viability. Under nutrient starvation or under environmental stresses, additional non-essential sigma factors (group 2 and group 3) compete for the limited amount of RNA polymerase core enzyme. Since the amino acid sequence of the DNA-binding region is conserved among group 1 and group 2 sigma factors, both sigma factors likely recognize similar promoter sequences. The group 3 sigma factors comprises alternative sigma factors that are structurally different from group 1 and group 2 sigma factors, and recognize promoters with a different consensus sequence and play a role in stress-induced transcription of specific regulons.

The chloroplast ancestor cyanobacteria also contain multiple sigma factors. The unicellular cyanobacterium Synechocystis sp. Strain PCC6803 possesses nine sigma factors; one group 1 (SigA), four group 2 and four group 3 sigma factors (Kaneko et al. 1996). The same is true for plastid sigma factors. In Arabidopsis, cDNAs encoding six different sigma factors have been isolated and sequenced (Isono et al. 1997; Tanaka et al. 1997; Fujiwara et al. 2000). Unlike cyanobacteria, all plastid sigma factors are related to group 1 and group 2 sigma factors and no group 3 sigma factor has been identified in plants. Phylogenetic analysis revealed that plastid sigma factors are grouped into five major sub groups (SIG1, SIG2, SIG3, SIG5 and SIG6). SIG5 is distinct from other plastid sigma factors in amino acid sequences and intron insertion sites (Fujiwara et al. 2000). Multiple plastid sigma factors may be involved in differential gene expression in response to developmental and environmental signals. The specificity of each sigma factor has recently been revealed by extensive characterization of Arabidopsis sigma factor T-DNA insertion mutants.

\section{Sigma factors responsible for early chloroplasts development}

Photosynthesis genes are not transcribed efficiently in seed plastids and their expression is up-regulated during germination and subsequent seedling development. It has been shown that SIG2 and SIG6 play crucial roles in early chloroplast development during light-dependent seedling growth in Arabidopsis. AtSIG6 T-DNA insertion mutants showed a pale green phenotype at an early stage of seedling development (3-5 days after imbibition: Figure 1), while the seedlings turn green at a later stage (7days) (Ishizaki et al. 2005; Loschelder, et al. 2006)). Light-dependent chloroplast development is significantly retarded in the AtSIG6 mutants. The transcript level of most PEP-dependent genes, including photosynthesis genes, rRNAs and some tRNAs are greatly decreased in young cotyledons by inactivation of SIG6, while NEPdependent transcripts mostly increased. All of the SIG6dependent genes are preceded by typical $\sigma^{70}$-type promoters. These data suggest that SIG6 is an early general sigma factor in plastids that plays a key role in chloroplast development at an early stage of seedling development. Detailed expression analysis of plastid sigma factors during germination revealed that SIG6 expression is transiently up-regulated between 1-3 days after imbibition, and then drop down. In addition to these early roles, it should be noted that SIG6 has the second (long term) role in mature leaves. Accumulation of 2.6 $\mathrm{kb}$ atpB/atpE transcripts was significantly reduced in SIG6 knock down mutants in mature leaves (Loschelder, et al. 2006).

SIG2-deficient mutants also showed a pale-green phenotype in Arabidopsis. However, unlike SIG6 mutants, the pale green phenotype has been observed in both young cotyledons and mature leaves (Figure 1), suggesting that SIG2 play a role in all stages of plant development. The pale green phenotype of AtSIG2deficeint mutants is characterized by decreased accumulation of chlorophyll and plastid-encoded photosynthesis related proteins (Kanamaru et al. 2001) However, most of the photosynthesis-related transcripts were not reduced in the AtSIG2-deficient mutants, suggesting that SIG2 is not responsible for transcription of plastid-encoded photosynthesis genes. On the other hand, the amount of several PEP-dependent tRNAs was significantly reduced in the AtSIG2-deficient mutants in both cotyledons and mature leaves. The SIG2-dependent 
A

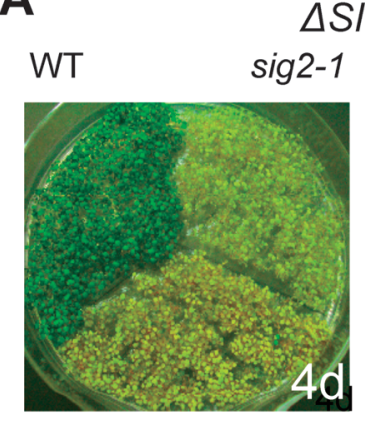

$\operatorname{sig} 2-2$

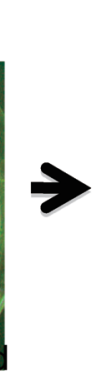

WT

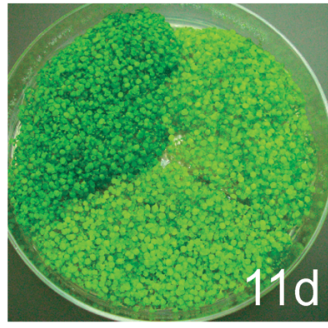

$\operatorname{sig} 2-2$ $\triangle S I G 2$ plants

B

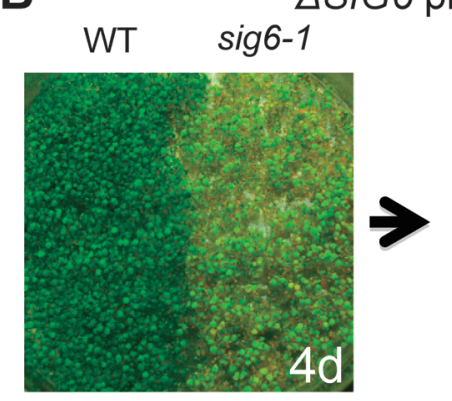

$\triangle$ SIG6 plants

WT sig6-1

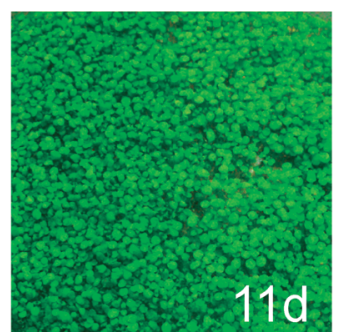

C

\begin{tabular}{|ll|}
\hline AtSIG1 & $\begin{array}{l}\text { Special target genes } \\
\text { OsSIG1 }\end{array}$ \\
& psaA operon \\
AtSIG2 & $\begin{array}{l}\text { tRNAs } \\
(t r n V, \text { trnM, trnE, trnD })\end{array}$ \\
AtSIG3 & $p s b N$ \\
AtSIG4 & $n d h F$ \\
AtSIG5 & $p s b D$ LRP \\
AtSIG6 & photosynthesis genes \\
\hline
\end{tabular}

Figure 1. Characterization of the Arabidopsis SIG2 and SIG6 mutants. (A) Wild-type (WT) and SIG2 T-DNA insertion mutants (sig2-1 and sig22). SIG2 mutants showed pale green phenotype in both young and older seedlings. (B) WT and SIG6 T-DNA insertion mutant (sig6-1). SIG6 mutants showed pale green phenotype in young seedlings, but the mutant phenotype was recovered in older seedlings. (C) Summary of functional diversity of plastid sigma factors in higher plants.

tRNAs include trnV-UAC, trnM-CAU, trnE-UUC and $\operatorname{trn} D$-GUC. It should be noted that $\operatorname{trn} E$ is a precursor of $5^{\prime}$-aminolevulinic acid (5'-ALA) and chlorophyll biosynthesis. Taken together, SIG2 is probably a special sigma factor that is indispensable for transcription of a set of tRNA genes. Decreased accumulation of photosynthesis related proteins without affecting their mRNA levels might be caused by defects in chlorophyll biosynthesis and/or plastid translation activity in the AtSIG2 mutants.

To date, SIG2, SIG3, SIG4, SIG5 and SIG6 T-DNA insertion mutants have been characterized in Arabidopsis. Only SIG2 and SIG6 T-DNA insertion mutants had pale green phenotypes in young cotyledons, whereas other mutants grew normally. Furthermore, detailed expression analysis of plastid sigma factors during seedling development in Arabidopsis revealed that SIG2 and SIG6 transcripts were detected within 1 day imbibition, while other sigma factor expression is induced after 2 days imbibition or later. The OSSIG6 (rice AtSIG6 homolog) transcripts was also more abundantly detected in the leaf base (young tissues) than in the leaf tip (matured tissue) (Kubota et al. 2007). Furthermore. The expression of maize ZmSIG6 protein was also detected in leaf base and root of light-grown seedlings (Lahiri and Allison, 2000). Taken together, these studies suggest that SIG6 and SIG2 cooperatively support the early transcription of photosynthesis genes and a set of tRNA genes during seedling development, respectively. Interestingly, SIG2/SIG6 double mutant shows an albino phenotype (Ishizaki et al. unpublished data). It is conceivable that coordinated functions of SIG6 and SIG2 in developing chloroplasts are essential for normal chloroplast development in Arabidopsis.

\section{Other General plastid sigma factors}

It should be noted that the AtSIG6-deficient mutant phenotype is restored in the later stages of seedling development (Ishizaki et al. 2005). It is assumed that another sigma factor(s) that recognizes the standard $\sigma^{70}$ type PEP promoters and is involved in the transcription of photosynthesis genes may take over the SIG6 functions in the later stage of seedling development. Since plastid sigma factors are related to group 1 and group 2 bacterial sigma factors that recognize $\sigma^{70}$-type promoter elements. it is expected that all plastid sigma factors can initiate transcription from the standard-type PEP promoters harboring -35 and -10 promoter elements. In fact, it has been demonstrated that $p s b A$ and $r b c L$ promoters are recognized by several sigma factors (Hakimi et al. 2000; Homann et al. 2003; Privat, 2003), suggesting overlapping specificity of plastid sigma factors.

On the other hand, it seems that plastid sigma factors 
have specific as well as overlapping functions. In vitro transcription assays performed without competition by other sigma factors may have masked the overlapping functions. In facts, the specific functions of plant sigma factors have been obtained by analyses of Arabidopsis TDNA insertion mutants that reflect in vivo competition conditions. For example, OsSIG1-deficient mutants have been characterized in rice (Tozawa et al. 2007). OsSIG1 mRNA accumulates relatively late during leaf development. The OsSIGI-deficient mutants showed a reduced chlorophyll content (one third). Moreover, transcripts derived from the plastid-encoded $p s a A$ operon were markedly decreased in the OsSIGI-deficient mutants. These results suggest that OsSIG1 is responsible for transcription of $p s a A$ operon genes for components of photosystem I in mature chloroplasts of rice. On the other hand, SIG1-deficient mutant has not been characterized in Arabidopsis.

On the other hand, AtSIG3-deficient mutants showed no visible phenotype (Zghidi et al. 2007). However, plastid genome microarray analysis revealed that $p s b N$ transcription was significantly reduced in the AtSIG3deficient mutants. The $p s b N$ transcription produces antisense RNA to the $p s b B$ operon. Thus, the SIG3dependent $p s b N$ transcription might serve a regulatory function for the expression of the $p s b B$ operon genes. AtSIG4-deficient mutants also showed no visible phenotype (Favory et al. 2005). It was shown that transcription of the plastid-encoded $n d h F$ gene was specifically reduced in the AtSIG4-deficient mutants. SIG4 may be involved in the regulation of the plastid $\mathrm{NDH}$ activity by regulating the $n d h F$ transcription.

In summary, SIG1, SIG3 and SIG4 likely have the specific functions in addition to the overlapping functions. It is conceivable that these sigma factors may support active transcription of photosynthesis genes in mature leaves together with SIG6, and may also be involved in the transcriptional regulation of specific genes in response to the developmental and environmental signals.

\section{Stress responsive sigma factor SIG5}

As mentioned above, the $p s b D$ light-responsive promoter ( $p s b D$ LRP) is the only promoter that is selectively activated by environmental stresses, including high salinity, high osmolarity, low temperature and high irradiance of blue light (Nagashima et al. 2004; Tsunoyama et al. 2002). Since the $p s b D$ LRP exhibits unusual promoter architecture $(p s b D$ LRP activity is dependent on the -10 element and AAG box, but not the -35 element), it is assumed that the $p s b D$ LRP might be recognized by a special sigma factor. Among higher plant sigma factors, AtSIG5 is structurally distinct from other sigma factors, and AtSIG5 expression is also specifically up-regulated by high-irradiance blue light
(Tsunoyama et al. 2002) and various environmental stresses (Nagashima et al. 2004). Analyses of Arabidopsis AtSIG5 T-DNA insertion mutants revealed that AtSIG5 is essential for the light-dependent $p s b D$ LRP activity (Tsunoyama et al. 2004; Nagashima et al. 2004). Moreover, over-expression of AtSIG5 in protoplasts (Tsunoyama et al. 2004) and in transgenic Arabidopsis and tobacco plants (Nozoe et al. unpublished data) resulted in the selective elevation of $p s b D$ LRP activity. Furthermore, high-intensity lightinduced $p s b D$ transcription was also dependent on the PpSIG5 in the moss Physcomitrella patensewas (Ichikawa et al. 2008). These findings demonstrated that SIG5 acts as a special sigma factor in chloroplasts that selectively recognizes the $p s b D$ LRP in response to various environmental stresses.

The germination rate of the AtSIG5 T-DNA insertion mutants decreased significantly under salinity conditions and the recovery process of PSII photochemical efficiency after exposure to high light was also delayed in the AtSIG5 mutants (Nagashima et al. 2004). On the other hand, over expression of AtSIG5 resulted in the higher germination rates in high salinity conditions (Figure 2; Kin et al. unpublished data). These data suggest that SIG5-dependent $p s b D$ LRP activation is directly or indirectly involved in the tress responses of Arabidopsis.

The expression of SIG5 is differentially induced by blue light but not red light (Tsunoyama et al. 2002) and the blue light-induced SIG5 expression is mediated by cryptochrome 1 and 2 (Mochizuki et al. 2004). The blue light-dependency of SIG5 expression is biphasic, the lower-light induction and higher-light induced further enhancement of SIG5 expression (Onda et al. 2008). Interestingly, the lower light induction is dependent on both cryptochrome 1 and 2, while stronger light dependent expression is mediated by only cryptochrome 1. On the other hand, SIG5, but not other sigma factors is quickly induced by red light in a Phytochrome-Adependent manner during de-etiolation of dark grown seedlings (Tepperman et al. 2006). Moreover, AtSIG5 TDNA insertion mutant displayed significant defects in the phytochrome-A-induced inhibition of hypocotyl elongation and stimulation of cotyledon growth, suggesting another direct or indirect role of SIG5 in the phytochrome-A-induced de-etiolation (Khannna et al. 2006). Furthermore, blue light-induced SIG5 expression is mediated by a nuclear localized protein phosphatase PP7 that has been identified as a positive regulator of blue light signaling (Müller et al. 2003). Finally, it should be noted that activation of the $p s b D$ LRP requires light even in the AtSIG5 overexpressing plants (Nozoe et al. unpublished data). The specific function of SIG5 beautifully illustrates how the nucleus holds control over plastid gene expression. Figure 2 represents a schematic 


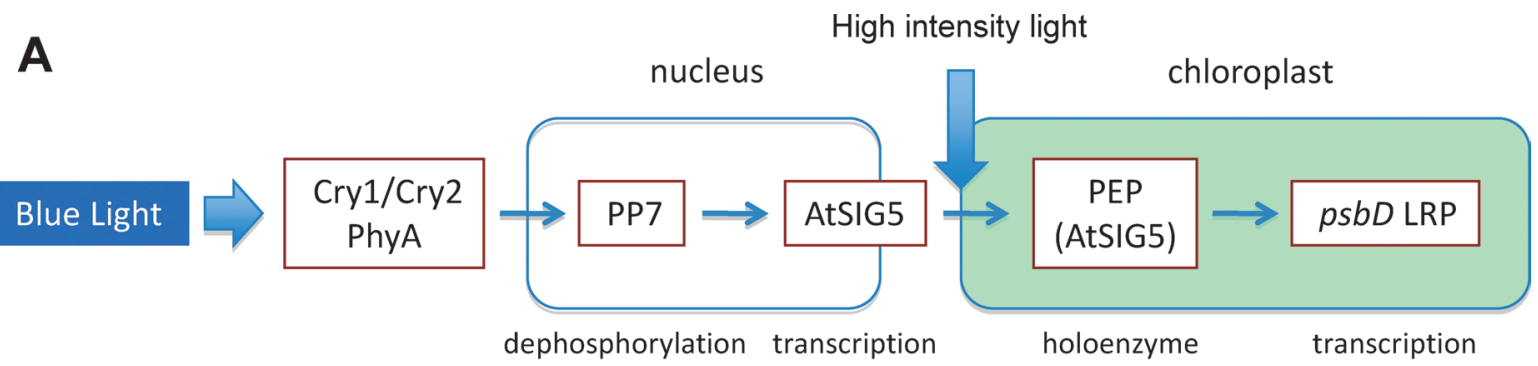

B
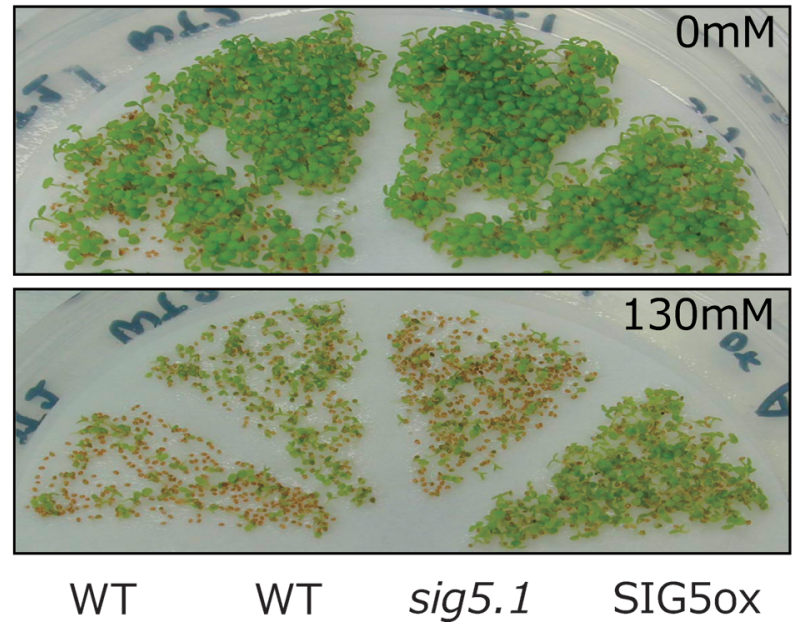

Figure 2. Characterization of the Arabidopsis SIG5 mutants. (A) Schematic representation of a possible mechanism of the bleu light-induced activation of $p s b D$ LRP. (B) Effects of high salinity on wild type (WT), SIG5 T-DNA insertion mutant (sig5.1) and SIG5 overexpressing mutant (SIG5Ox) plants. The germination rate of the SIG5-defieicnt mutants decreased significantly, while over expression of SIG5 resulted in the higher germination rates in high salinity conditions.

model of SIG5-mediated activation of $p s b D$ LRP.

\section{Post-transcriptional regulation of plastid sigma factors}

There is some evidence to suggest that plant sigma factors might be regulated post-translationally. SIB1 is a nuclear-encoded SIG1-binding protein that targets to chloroplasts. The activity of SIG1 might be controlled by its interaction with SIB1 (Morikawa et al. 2002). On the other hand, the activity of SIG3 might be regulated by proteolytic cleavage (Hkimi et al. 2000; Hoffmann et al. 2003). Furthermore, biochemical evidence suggests that plastid sigma factors might be regulated by protein phosphorylation in mustard (Baginsky et al. 1997)

\section{Evolution of plastid sigma factors}

As mentioned above, the cyanobacterial ancestor of the plastids has group 3 sigma factors, besides group 1 and group 2 sigma factors. On the other hand, no sigma factor of the group 3 has been found in plant genomes. Primitive red algae Cyanidioschyzon merolae genome encodes four plastid sigma factors (CmSigl-4) (Matsuzaki et al. 2005), while green algae Chlamydomonas reinghardii encodes a single sigma factor (CrRpoD) (Bohne et al. 2006). Phylogenetic analysis of plastid sigma factors suggested that plant sigma factors of red and green algal plastids, and the group 1 sigma factors of cyanobacteria form a monophyletic group (Minoda et al. 2005). Considering monophyletic nature of land plants and green algae, it is also likely that all plastid sigma factors are derived from cyanobacterial group1 (principal) sigma factor. It is assumed that the principal sigma factor genes were transferred from the ancestral plastid to the host nuclear genome. Subsequently, the nuclear encoded principal sigma factors were diversified during plant evolution. Figure 3 shows the possible evolution of plastid sigma factors in higher plants.

There are four conserved intron insertion sites in higher plant sigma factors, except for SIG5. These introns are not conserved in green algal (CrRpoD) and red algal (CmSig1-4) sigma factors. Thus, it is assumed that the sigma factors of green and red algae were evolved independently from higher plant sigma factors. Moreover, the amino acid sequence of SIG5 is different from other plastid sigma factors in higher plants, suggesting that Sig5 was firstly diverged from the common ancestral plastid sigma factor and evolved 


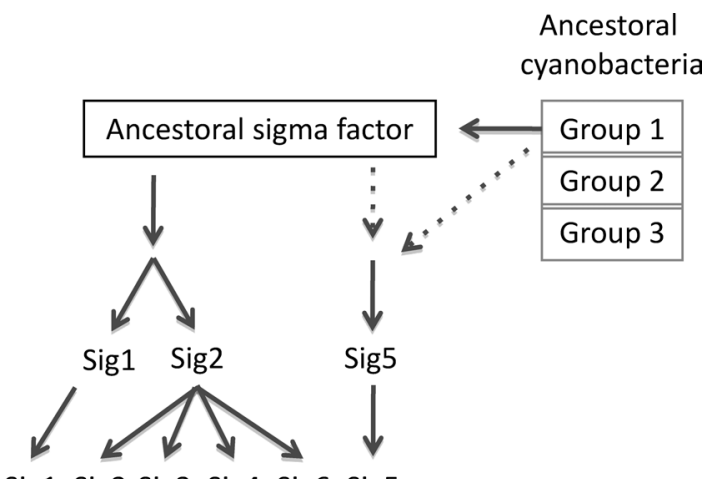

Sig1 Sig2 Sig3 Sig4 Sig6 Sig5

Figure 3. Schematic representation of the plastid sigma factor gene evolution. Dotted lines represent unidentified route of Sig 5 origin.

independently. Furthermore, the analysis of intron-exon structure suggested that the common ancestor of higher plant sigma factors first duplicated into SIG1 and SIG2. In facts, the moss Physcomitrella patens (Rensing et al. 2008) and early divergent vascular land plant Selaginella nvolvens (see Selaginella Genomics: http://selaginella. genomics.purdue.edu/) has SIG1, SIG2 and SIG5 homologs, but not SIG3, SIG4 and SIG6. These ancient type sigma factors may have emerged in Streptophyta phylum, the common ancestors of charophytes and higher plants. Subsequently SIG3 and SIG6 likely emerged from $S I G 2$ during the evolution of angiosperms, before the separation of monocots and dicots. Finally AtSIG4 was evolved in dicots after the separation of monocots and dicots, since SIG4 homolog was not found in the rice genome. On the other hand, four sigma factors in Cyanidioschyzon merolae probably evolved independently in primitive red algae.

A sharp increase in diversity of plastid sigma factors may be involved in the increasing complexity of plant body plans and meat the increasing demand for stress tolerance during evolution of higher plants. Further detailed functional and expression analysis of each sigma factor will shed light on the role of multiple plastid sigma factors in higher plants.

\section{Acknowledgements}

We thank Satoko Kin and Shu-ichi Shinmura for providing us unpublished data. This work was supported by a grant to TS from the New Energy and Industrial Technology Development Organization (NEDO) and also supported by Grant in-Aid to YY for Scientific Research for Plant Graduate Student from Nara Institute Science and Technology, Supported by The Ministry of Education, Culture, Sports, Science and Technology, JAPAN.

\section{References}

Baba K, Nakano T, Yamagishi K, Yoshida S (2001) Involvement of a nuclear-encoded basic helix-loop-helix protein in transcription of the light-responsive promoter of psbD. Plant Physiol 125:
595-603

Baginsky S, Tiller K, Pfannschmidt T, Link G (1997) PTK, the chloroplast RNA polymerase-associated protein kinase from mustard (Sinapis alba), mediates redox control of plastid in vitro transcription. Plant Mol Biol 39: 1013-1023

Bohne AV, Irihimovitch V, Weihe A, Stern DB (2006) Chlamydomonas reinhardtii encodes a single sigma70-like factor which likely functions in chloroplast transcription. Curr Genet 49: 333-340

Chang CC, Sheen J, Niwa Y, Lerbs-Mache S, Stern DB (1999) Functional analysis of two Maize cDNAs encoding T7-like RNA polymerases. Plant Cell 11: 911-926

Christopher DA, Mullet JE (1994) Separate photosensory pathways co-regulate blue light/ultraviolet-A-activated $p s b D-p s b C$ transcription and light-induced D2 and CP43 degradation in barley (Hordeum vulgare) chloroplasts. Plant Physiol 104: 1119-1129

Chun L, Kawakami A, Christopher DA (2001) Phytochrome A mediates blue light and UV-A-dependent chloroplast gene transcription in green leaves. Plant Physiol 125: 1957-1966

Courtois F, Merendino L, Demarsy E, Mache R, Lerbs-Mache S (2007) Phage-type RNA polymerase RPOTmp transcribes the rrn operon from the PC promoter at early developmental stages in Arabidopsis. Plant Physiol 145: 712-721

Demarsy E., Courtois F., Azvedo J., Buhot L, Lerbs-Mache S (2006) Building up of the plastid transcriptional machinery during germination and early plant development. Plant Physiol 142: 993-1003

Eisermann A, Tiller K, Link G (1990) In vitro transcription and DNA binding characteristics of chloroplast and etioplast extracts from mustard (Sinapis alba) indicate differential usage of the psbA promoter. EMBO J 9: 3981-3987

Emanuel C, von Groll U, Müller M, Börner T, Weihe A (2006) Development- and tissue-specific expression of the RpoT gene family of Arabidopsis encoding mitochondrial and plastid RNA polymerases. Planta 223: 998-1009

Favory JJ, Kobayshi M, Tanaka K, Peltier G, Kreis M, Valay JG, Lerbs-Mache S (2005) Specific function of a plastid sigma factor for $n d h F$ gene transcription. Nucl Acids Res 33: 5991-5999

Fujiwara M, Nagashima A, Kanamaru K, Tanaka K, Takahashi H (2000) Three new nuclear genes, sigD, sigE and sigF, encoding putative plastid RNA polymerase factors in Arabidopsis thaliana FEBS Lett 481: 47-52

Funk HT, Berg S, Krupinska K, Maier UG, Krause K (2007) Complete DNA sequences of the plastid genomes of two parasitic flowering plant species, Cuscuta reflexa and Cuscuta gronovii. BMC Plant Biol 22: 45

Hakimi MA, Privat I, Valay JG, Lerbs-Mache S (2000) Evolutionary conservation of C-terminal domains of primary sigma70-type transcription factors between plants and bacteria. J Biol Chem 275: 9215-9221

Hajdukiewicz PTJ, Allison LA, Maliga P (1997) The two RNA polymerases encoded by the nuclear and the plastid compartments transcribe distinct groups of genes in tobacco plastids. EMBO J 16: 4041-4048

Hedtke B, Börner T, Weihe A (2000) One RNA polymerase serving two genomes. EMBO Rep 1: 435-440

Hess WR, Börner T (1999) Organellar RNA polymerases of higher plants. Int Rev Cytol 190: 1-59

Homann A, Link G (2003) DNA-binding and transcription characterization of three cloned sigma factors from mustard 
(Sinapis alba L.) suggest overlapping and distinct roles in plastid gene expression. Eur J Biochem 270: 1288-1300

Ichikawa K, Shimizu A, Okada R, Satbhai SB, Aoki S (2006) The plastid sigma factor SIG5 is involved in the diurnal regulation of the chloroplast gene $p s b D$ in the moss Physcomitrella patens. FEBS Lett 582: 405-409

Ishizaki Y, Tsunoyama Y, Hatano K, Ando K, Kato K, Shinmyo A, Kobori M, Takeba G, Nakahira Y, Shiina T (2005) A nuclearencoded sigma factor, Arabidopsis SIG6, recognizes sigma-70 type chloroplast promoters and regulates early chloroplast development in cotyledons. Plant J 42: 133-144

Isono K, Shimizu M, Yoshimoto K, Niwa Y, Satoh K, Yokota A, Kobayashi H (1997) Leaf-specific expressed genes for polypeptides destined for chloroplasts with domains of $\sigma^{70}$ factors of bacterial RNA polymerases in Arabidopsis thaliana Proc Natl Acad Sci USA 94: 14948-14953

Kabeya Y, Hashimoto K, Sato N (2002). Identification and characterization of two phage-type RNA polymerase cDNAs in the moss Physcomitrella patens: Implication of recent evolution of nuclear-encoded RNA polymerase of plastids in plants. Plant Cell Physiol 43: 245-255

Kanamaru K, Nagashima A, Fujiwara M, Shimada H, Shirano Y, Nakabayashi K, Shibata D, Tanaka K, Takahashi H (2001) An Arabidopsis sigma factor (SIG2)-dependent expression of plastid-encoded tRNAs in chloroplasts. Plant Cell Physiol 42: 1034-1043

Kanamaru K, Tanaka K (2004) Roles of chloroplast RNA polymerase sigma factors in chloroplast development and stress response in higher plants. Biosci Biotechnol Biochem 68: 2215-2223

Kaneko T, Sato S, Kotani H, Tanaka A, Asamizu E, Nakamura Y, Miyajima N, Hirosawa M, Sugiura M, Sasamoto S, et al (1996) Sequence analysis of the genome of the unicellular cyanobacterium Synechocystis sp. strain PCC 6803. II. Sequence determination of the entire genome and assignment of potential protein-coding regions. DNA Res 3: 109-136

Kim M, Thum KE, Morishige DT, Mullet JE (1999) Detailed architecture of the barley chloroplast $p s b D-p s b C$ blue lightresponsive promoter. J Biol Chem 274: 4684-4692

Kim M, Mullet JE (1995) Identification of a sequence-specific DNA binding factor required for transcription of the barley chloroplast blue light-responsive $p s b D-p s b C$ promoter. Plant Cell 7: 1445-1457

Khanna R, Shen Y, Toledo-Ortiz G, Kikis EA, Johannesson H, Hwang YS, Quail PH (2006) Functional profiling reveals that only a small number of phytochrome-regulated early-response genes in Arabidopsis are necessary for optimal deetiolation. Plant Cell 18: 2157-2171

KodamaY, Sano H (2007) A comparative analysis of basic helix-loop-helix proteins, AtPTF1 and NtWIN4, with reference to plastid localization. Plant Biotechnol 403: 493-499

Kubota Y, Miyao A, Hirochika H, Tozawa Y, Yasuda H, Tsunoyama Y, Niwa Y, Imamura S, Shirai M, Asayama M (2007) Two novel nuclear genes, OsSIG5 and OsSIG6, encoding potential plastid sigma factors of RNA polymerase in rice: tissue-specific and light-responsive gene expression. Plant Cell Physiol 48: 186-192

Lahiri SD, Allison LA (2000) Complementary expression of two plastid-localized sigma-like factors in maize. Plant Physiol 123: 883-894

Lonetto M, Gribskov M, Gross CA. (1992) The $\sigma^{70}$ family: sequence conservation and evolutionary relationships. $J$
Bacteriol 174: 3843-3849

Loschelder H, Schweer J, Link B, Link G (2006) Dual temporal role of plastid sigma factor 6 in Arabidopsis development. Plant Physiol 142: 642-650

Matsuzaki M, Misumi O, Shin-I T, Maruyama S, Takahara M, et al (2008) Genome sequence of the ultrasmall unicellular red alga Cyanidioschyzon merolae 10D. Nature 428: 653-657

Matsuo M, Ito Y, Yamauchi R, Obokata J (2005) The rice nuclear genome continuously integrates, shuffles, and eliminates the chloroplast genome to cause chloroplast-nuclear DNA flux. Plant Cell 17: 665-675

Minoda A, Nagasawa K, Hanaoka M, Horiuchi M, Takahashi H, Tanaka K (2005) Microarray profiling of plastid gene expression in a unicellular red alga, Cyanidioschyzon merolae. Plant Mol Biol 59: 375-385

Müller SG, Kim YS, Kunkel T, Chua NH (2003) PP7 is a positive regulator of blue light signaling in Arabidopsis. Plant Cell 15: 1111-1119

Morikawa K, Shiina T, Murakami S, Toyoshima Y (2002) Novel nuclear-encoded proteins interacting with a plastid sigma factor, Sig1, in Arabidopsis thaliana. FEBS Lett 514: 300-304

Mochizuki T, Onda Y, Fujiwara E, Wada M, Toyoshima Y (2004) Two independent light signals cooperate in the activation of the plastid psbD blue light-responsive promoter in Arabidopsis. FEBS Lett 571: 26-30

Mullet JE (1993) Dynamic regulation of chloroplast transcription. Plant Physiol 103: 309-313

Nagashima A, Hanaoka M, Shikanai T, Fujiwara M, Kanamaru K, Takahashi H, Tanaka K (2004) The multiple-stress responsive plastid sigma factor, SIG5, directs activation of the $p s b D$ blue light-responsive promoter (BLRP) in Arabidopsis thaliana. Plant Cell Physiol 45: 357-368

Nakahira Y, Baba K, Yoneda A, Shiina T, Toyoshima Y (1998) Circadian-regulated transcription of the $p s b D$ light-responsive promoter in wheat chloroplasts. Plant Physiol 118: 1079-1088

Nakamura Y, Kaneko T, Hirosawa M, Miyajima N, Tabata S (1998) CyanoBase, a www database containing the complete nucleotide sequence of the genome of Synechocystis sp. strain PCC6803. Nucl Acids Res 26: 63-67

Onda Y, Yagi Y, Saito Y, Takenaka N, Toyoshima Y (2008) Light induction of Arabidopsis SIG1 and SIG5 transcripts in mature leaves: differential roles of cryptochrome 1 and cryptochrome 2 and dual function of SIG5 in the recognition of plastid promoters. Plant J 55: 968-978

Privat I, Hakimi M-A, Buhot L, Favory JJ. Lerbs-Mache S (2003) Characterization of Arabidopsis plastid sigma-like transcription factors SIG1, SIG2 and SIG3. Plant Mol Biol 55: 385-399

Rensing SA, Lang D, Zimmer AD, Terry A, Salamov A, et al (2008) The Physcomitrella genome reveals evolutionary insights into the conquest of land by plants. Science 319: 64-69

Sato N (2001) Was the evolution of plastid genetic machinery discontinuous? Trends Plant Sci 6: 151-155

Sato S, Nakamura Y, Kaneko T, Asamizu E, Tabata S (1999) Complete structure of the chloroplast genome of Arabidopsis thaliana. DNA Res 65: 283-290

Satoh J, Baba K, Nakahira Y, Tsunoyama Y, Shiina T, Toyoshima Y (1999) Developmental stage-specific multi-subunit plastid RNA polymerases (PEP) in wheat. Plant J 18: 407-415

Sriraman P, Silhavy D, Maliga P (1998) The phage-type PclpP-53 plastid promoter comprises sequences downstream of the transcription initiation site. Nucl Acids Res 26: 4874-4879

Suzuki JY, Sriraman P, Svab Z, Maliga P (2003) Unique 
architecture of the plastid ribosomal RNA operon promoter recognized by the multisubunit RNA polymerase in tobacco and other higher plants. Plant Cell 15: 195-205

Swiatecka-Hagenbruch M, Emanuel C, Hedtke B, Liere K, Börner $\mathrm{T}$ (2008) Impaired function of the phage-type RNA polymerase RpoTp in transcription of chloroplast genes is compensated by a second phage-type RNA polymerase. Nucl Acids Res 36: 785-792

Tanaka K, Tozawa Y, Mochizuki N, Shinozaki K, Nagatani A, Wakasa K, Takahashi H (1997) Characterization of three cDNA species encoding plastid RNA polymerase sigma factors in Arabidopsis thaliana: evidence for the sigma factor heterogeneity in higher plant plastids. FEBS Lett 413: 309-313

Tepperman JM, Hwang Y-S, Quail PH (2006) phyA dominates in transduction of red-light signals to rapidly-responding genes at the initiation of Arabidopsis seedling deetiolation. Plant J 48: $728-742$

Thum KE, Kim M, Christopher DA, Mullet JE (2001) Cryptochrome 1, cryptochrome 2, and phytochrome a coactivate the chloroplast $p s b D$ blue light-responsive promoter. Plant Cell 13: 2747-2760

To KY, Cheng MC, Suen DF, Mon DP, Chen LF, Chen SC (1996) Characterization of the light-responsive promoter of rice chloroplast $p s b D-C$ operon and the sequence-specific DNA binding factor. Plant Cell Physiol 37: 660-666

Tozawa Y, Teraishi M, Sasaki T, Sonoike K, Nishiyama Y, Itaya M, Miyao A, Hirochika H (2007) The plastid sigma factor SIG1 maintains photosystem I activity via regulated expression of the psaA operon in rice chloroplasts. Plant J 52: 124-132

Tsunoyama Y, Ishizaki Y, Morikawa K, Kobori M, Nakahira Y, Takeba G, Toyoshima Y, Shiina T (2004) Blue light-induced transcription of plastid-encoded $p s b D$ gene is mediated by a nuclear-encoded transcription initiation factor, AtSig5. Proc Natl Acad Sci USA 101: 3304-3309

Tsunoyama Y, Morikawa K, Shiina T, Toyoshima Y. (2002) Blue light specific and differential expression of a plastid sigma factor, Sig5 in Arabidopsis thaliana. FEBS Lett 516: 225-228

Wolfe KH, Morden CW, Palmer JD (1992) Function and evolution of a minimal plastid genome from a nonphotosynthetic parasitic plant. Proc Natl Acad Sci USA 89: 10648-10652

Zghidi W, Merendino L, Cottet A, Mache R, Lerbs-Mache S. (2007) Nucleus-encoded plastid sigma factor SIG3 transcribes specifically the psbN gene in plastids. Nucl Acids Res 35: $455-64$

Zybailov B, Rutschow H, Friso G, Rudella A, Emanuelsson O, Sun Q, van Wijk KJ. (2008) Sorting signals, N-terminal modifications and abundance of the chloroplast proteome. PLOS ONE 3, e1994 